\title{
Development and analysis of an adverse outcome pathway network for human neurotoxicity
}

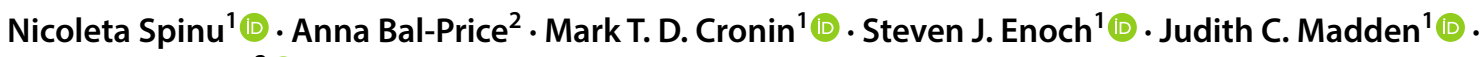 \\ Andrew P. Worth ${ }^{2}$ (B)
}

Received: 15 June 2019 / Accepted: 14 August 2019 / Published online: 23 August 2019

(c) The Author(s) 2019

\begin{abstract}
An adverse outcome pathway (AOP) network is an attempt to represent the complexity of systems toxicology. This study illustrates how an AOP network can be derived and analysed in terms of its topological features to guide research and support chemical risk assessment. A four-step workflow describing general design principles and applied design principles was established and implemented. An AOP network linking nine linear AOPs was mapped and made available in AOPXplorer. The resultant AOP network was modelled and analysed in terms of its topological features, including level of degree, eccentricity and betweenness centrality. Several well-connected KEs were identified, and cell injury/death was established as the most hyperlinked KE across the network. The derived network expands the utility of linear AOPs to better understand signalling pathways involved in developmental and adult/ageing neurotoxicity. The results provide a solid basis to guide the development of in vitro test method batteries, as well as further quantitative modelling of key events (KEs) and key event relationships (KERs) in the AOP network, with an eventual aim to support hazard characterisation and chemical risk assessment.
\end{abstract}

Keywords Adverse outcome pathway $\cdot$ Predictive toxicology $\cdot$ Network development $\cdot$ Network analytics $\cdot$ Neurotoxicity

\section{Introduction}

The science of networks is defined as the collection, management, analysis, interpretation and presentation of relational data (Brandes et al. 2013). The investigation of networks is spread widely throughout all branches of biology and chemistry, from neurobiology (Bassett and Sporns 2017) to genomics (Li et al. 2017). For example, in biology, the application of networks has made advances towards uncovering the organising principles of various complex systems, e.g. protein-protein interactions, metabolomics, signalling and transcription-regulatory networks (Barabasi and Oltvai

Electronic supplementary material The online version of this article (https://doi.org/10.1007/s00204-019-02551-1) contains supplementary material, which is available to authorized users.

Andrew P. Worth

andrew.worth@ec.europa.eu

1 School of Pharmacy and Biomolecular Sciences, Liverpool John Moores University, Byrom Street, Liverpool L3 3AF, UK

2 European Commission, Joint Research Centre (JRC), Ispra, Italy
2004). On the other hand, systems toxicology, considered as an application of systems biology, aims to describe the perturbation by toxicants and the resilience of the essential defence and adaptive mechanisms across multiple levels of biological organisations (Hartung et al. 2017; Sturla et al. 2014). In other words, systems toxicology helps to identify meaningful disease-specific biomarkers as opposed to systems biology where the purpose is to discover the underlying molecular and cellular mechanisms (Aguayo-Orozco et al. 2019). Systems biology captures interactions between biological entities, while systems toxicology focuses on the temporal/spatial relationships between processes/ events, triggered by an exposure to stressor(s), particularly chemicals.

Following the publication of the National Research Council's report on "Toxicity Testing in the 21st Century" (Krewski et al. 2010), there was an increased interest in using information from physiological pathways, and their perturbation, to better inform the adverse effects of chemicals. In many ways, this provided the stimulus to develop the adverse outcome pathway (AOP) concept (Ankley et al. 2010) into a systematic framework to collect and organise mechanistic knowledge in the field of predictive toxicology. An AOP 
is a linear description of a toxicological process from the molecular initiating event (MIE), in which a stressor first perturbs the biological system, through a series of intermediate or key events (KEs), to the adverse outcome (AO) that may manifest as a consequence. In recent years, AOPs have become a tool to support the development and application of in vitro and in silico testing strategies for the assessment of adversity while offering new insights into in vivo outcomes of regulatory interest (Kleinstreuer et al. 2016).

Although AOPs are linear constructs and thus a simplification of complex physiological and toxicological processes (Vinken et al. 2017), it is well appreciated that AOPs are interconnected and potentially share the same processes or key events (Knapen et al. 2018). As such, network science provides an appealing framework to better represent the complexity of biological processes by studying relationships among interconnected linear AOPs. The term "AOP network" can be defined as a set of individual AOPs sharing at least one common element represented by a KE, including an MIE and an AO (Villeneuve et al. 2014a). Different AOPs diverging from a single MIE, or converging to a single $\mathrm{AO}$, also form AOP networks, even if they do not have any other $\mathrm{KE}$ in common (Knapen et al. 2018). An individual AOP can be considered as a pragmatic unit of development and evaluation, while an AOP network can be seen as the functional unit of prediction (Villeneuve et al. 2014a, b). Hence, an individual AOP should be treated as a building block within a larger AOP network that more comprehensively describes the biological processes involved in real-world scenarios. This does, however, imply that it will become increasingly important to move away from viewing single linear AOPs in isolation and to consider instead non-linear and branched AOPs within the broader context of AOP networks, as acknowledged in recent guidance (OECD 2017). The challenge is to integrate individual AOPs into a network for a predefined application and to characterise the network in quantifiable terms.

The Organisation for Economic Co-operation and Development (OECD) AOP Knowledge Base (AOP-KB) project, especially the AOP-Wiki module (https://aopwiki.org/), brings together the scientific community to develop, share and discuss AOP-related knowledge while accelerating and facilitating AOP development in a central location, allowing the connectivity of AOPs to be explored (Villeneuve et al. 2014a). By developing an AOP network, all the possible AOPs in the AOP-Wiki that are relevant to the specific question may be examined. As AOPs are living documents (Villeneuve et al. 2014a), capable of accommodating updates to the description of key event relationships (KERs) and the addition of new KEs, AOP networks should also be regarded as living documents. The AOP-Wiki module is designed to automatically generate AOP networks through the identification of common KEs involved in multiple AOPs. This allows information that has been curated for one AOP to be reused in another, avoiding duplication of effort. Recently, a "global" AOP network was developed to evaluate the overall connectivity and structural features of existing linear AOPs in the AOP-Wiki module (Pollesch et al. 2019). This illustrated the possibility of deriving AOP networks for toxicological applications. However, the development and use of AOP networks are still in its infancy, and further proofof-concept examples are needed, including approaches for characterising the underlying uncertainties and limitations (Edwards et al. 2016).

To describe and analyse an AOP network, a range of network analytics can be used to identify and investigate specific network properties, such as topological features or interactions between linear AOPs (Knapen et al. 2018). Although the visual examination of the AOP network graph is compelling, the use of techniques from graph theory facilitates the interpretation of a network in terms of its quantitative topological characteristics. To analyse the topology of an AOP network, many metrics can be calculated to describe the overall shape and structure of the network. Several parameters were identified and described by Villeneuve et al. (2018a), such as level of degree (also known as valency, here the number of KERs linked to a KE), betweenness centrality, path occurrence, eccentricity, topological sorting, connectivity, contraction and matching index. Using these kinds of metrics helps to identify the most upstream or downstream KEs, points of convergence and divergence, positive and negative feedback loops, etc. The topological parameters allow not only the characterisation of AOP networks, but also the identification of the most common/highly connected KEs. In the absence of empirical information on the toxicological relevance of individual KEs, it seems reasonable to priortise the most highly connected KEs for testing and quantification. These topological parameters can be grouped further depending on the question of interest and network size, so that not all of them need to be measured at once. For example, an AOP network can be tested for degree, path occurrence, betweenness centrality and eccentricity in order to identify points of convergence and/or divergence. Thus, a key challenge is how to establish which parameters are most relevant for the specific question and context of use.

Developmental and adult/ageing neurotoxicity are important endpoints in chemical risk assessment and are emerging fields for method development and use in regulatory decision making (Bal-Price et al. 2018a; Fritsche et al. 2018). Early life exposures to certain chemicals, such as pesticides, may have long-term adverse health consequences for the developing brain. In addition, adult/ageing neurotoxicity, e.g. Alzheimer's and Parkinson's diseases, pose major challenges for societies with rapidly ageing populations. Various test systems are used to evaluate the neurotoxicity of a chemical, including cell lines, primary rodent cells, induced 
pluripotent stem cells-derived mixed neuronal/glial cultures in 2D and 3D cultures, etc. (Bal-Price et al. 2015a; Schmidt et al. 2017). While none of these is currently validated for regulatory use (e.g. as OECD Test Guidelines), they provide relevant information for AOP development (Bal-Price et al. 2015b). Furthermore, evaluating and mapping available linear AOPs for neurotoxicity into a network help to understand the causative linkages between KEs in terms of mechanistic knowledge supported by empirical evidence, while identifying knowledge gaps, limitations and opportunities related to pathophysiological pathways involved.

The main aim of this study was to develop an AOP network for human neurotoxicity and characterise the network using the analytics proposed by Knapen et al. (2018) and Villeneuve et al. (2018a). We also formulate and utilise a workflow to guide scientists interested in the development of AOP networks. In addition, a further aim was to analyse the neurotoxicity AOP network to identify the most common/ highly connected KEs and KERs as the basis for quantitative modelling.

\section{Materials and methods}

\section{Data Set}

Linear AOPs from the OECD AOP-Wiki 2.0 were investigated manually to develop the derived AOP network following the criteria described in "Network construction". The following information about the status of individual AOPs was extracted and collected in an Excel spreadsheet available as supplementary information: progress through the OECD review and endorsement processes (e.g. under development, endorsement by the Working Party on Hazard Assessment (WPHA)/Working Group of the National Coordinators of the Test Guidelines Programme (WNT), approval of the Extended Advisory Group on Molecular Screening and Toxicogenomics (EAGMST)), KE title, KE type (i.e. MIE, KE, AO), KER (i.e. linkage between upstream and downstream KEs), adjacency of the relationship between a pair of KEs, and qualitative weight of evidence. The linear AOPs were collected in December 2018 and the last check was performed in July 2019.

\section{Stressors}

The OECD AOP-Wiki 2.0 was also used to extract the stressors (chemical initiators and/or non-chemical stressors) triggering KEs, including MIEs and AOs of the collected linear AOPs together with the available unique identifier number used in PubMed (PMIDs) listed in the stressor's description page. The data for the stressors (in this case, all chemicals) were compiled in an Excel spreadsheet. In addition, the
Chemical Abstracts Service Registry Number (CAS RN), Simplified Molecular Input Line Entry System (SMILES) strings, and details on the industrial and therapeutic uses were retrieved from the PubChem database (https://pubch em.ncbi.nlm.nih.gov/) to understand the nature of the chemical stressor responsible for the initiation of the linear AOPs. At the same time, the ToxCast ${ }^{\mathrm{TM}}$ Dashboard (https://actor .epa.gov/dashboard/) was investigated for relevant data relating to assays that may be associated with the KEs, including MIEs and AOs of the collected linear AOPs.

\section{Network construction}

The process of developing a so-called "derived" AOP network (i.e. derived from existing AOPs) followed the four steps that are illustrated in Fig. 1. Initially, the "General Design Principles" were formulated. These principles are intended to be generic in nature and can be applied to any other question of interest. The "Applied Design Principles" are an exemplified version of the General Design Principles followed for the development of an organ specific AOP network-in Fig. 1, this is illustrated for neurotoxicity. The methodology of each part of the four-step process is described below.

\section{Step 1}

Step 1 of the workflow is the definition of the purpose of an AOP network to be modelled. In this investigation, the purpose was to identify the most common/highly connected KEs and KERs in neurotoxicity AOP network as the basis for quantitative modelling. Accordingly, the scope of the exercise included the linear AOPs known for human neurotoxicity formulated and published in the AOP-Wiki module.

\section{Step 2}

Step 2 of the workflow is the definition of the criteria for the selection of the AOPs for the development of the network. In this investigation, the criteria included:

- the AOP development stage in terms of the progress of the AOP through the OECD review and endorsement processes,

- the life stage applicability, and

- the taxonomic applicability.

Of these, taxonomy was chosen to be the main criterion for collecting individual AOPs, i.e. those for human toxicology. The AOP development stage was investigated to evaluate the level of maturity of the AOPs used to derive the AOP network. It gives an indication of uncertainties 


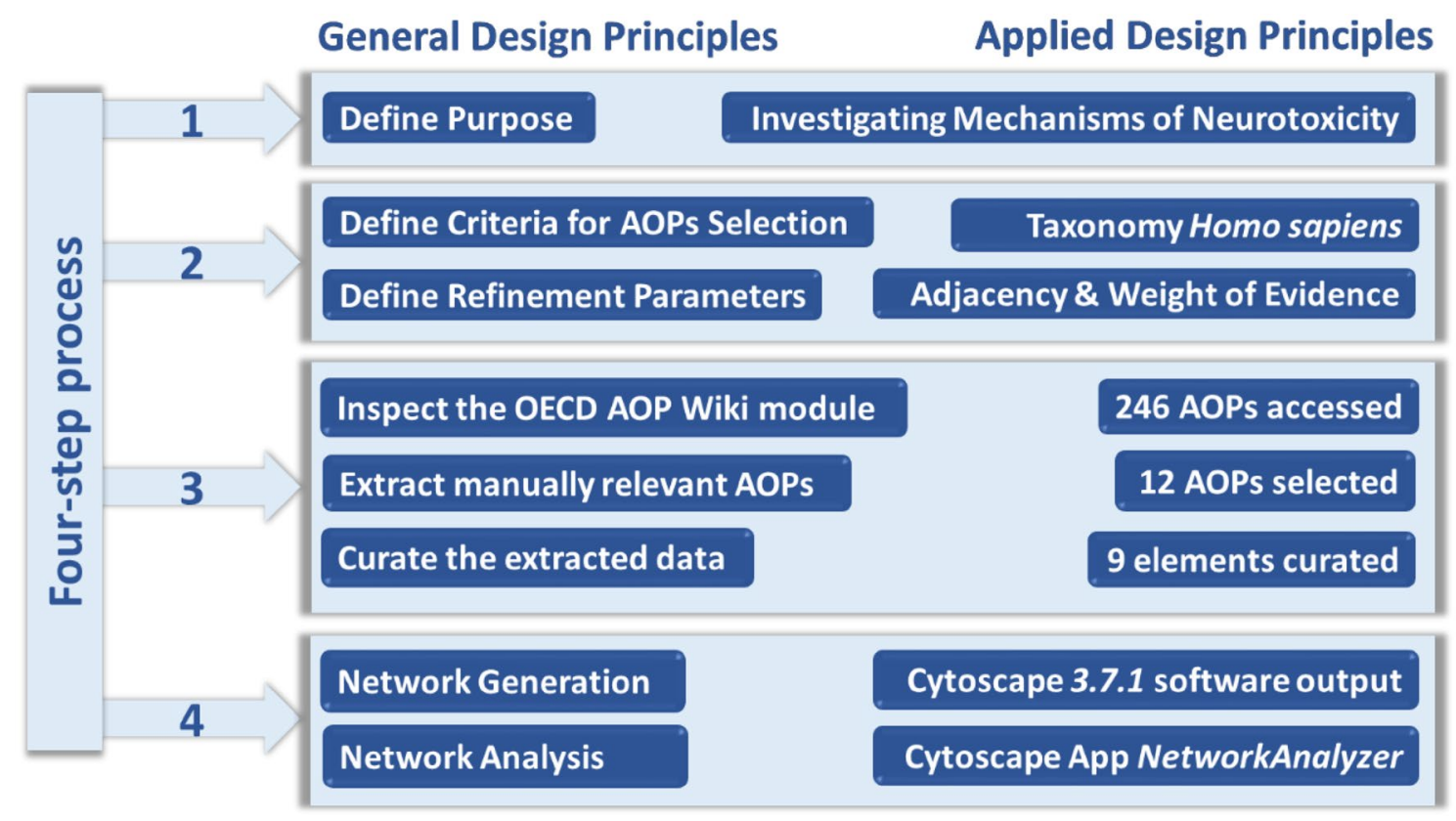

Fig. 1 The general design principles of the four-step workflow for developing a derived AOP network illustrated by applied design principles with regard to human neurotoxicity

in the AOP network and shows where further efforts are needed to elucidate the underlying mechanisms.

As one of the uses of an AOP network is the quantitative modelling, developing a network with a high level of qualitative and quantitative evidence will give confidence to the model applicability. As such, refinement of the parameters for the initially collected linear AOPs following Step 1 was considered: adjacency and non-adjacency of relationships between a pair of KEs, and the weight of evidence, specifically the qualitative level of understanding for the relationships between a pair of KEs: high, medium or low. Considerations of the Bradford Hill criteria for weight of evidence assessment were out of scope of this modelling exercise. This study relied on the assessments performed by the authors of the AOPs which are summarised in dedicated tables in the AOP-Wiki.

\section{Step 3}

Step 3 of the workflow is the identification of appropriate AOPs from the AOP-Wiki module. The AOPs identified according to the criteria in Step 2 were inspected and collected manually in an Excel spreadsheet. The information contained in the AOPs was subsequently curated using the ontology annotations of KEs titles.

\section{Step 4}

Step 4 of the workflow is the generation and analysis of the network. Cytoscape 3.7.1 (https://cytoscape.org/), an open source software platform, was used to model the AOP network, and NetworkAnalyzer 3.3.2 App (Assenov et al. 2008), a pre-installed application of the Cytoscape software, was used to analyse the resulting AOP network. The nodes were manually positioned as needed to conserve space and maximise readability. Additional annotation information (e.g. weight of evidence, adjacency and type of KE) was used to further define the visual attributes of the AOP network. The KEs shared by more than one AOP are shown graphically as nonrepetitive (i.e. represented by a single arrow), while the duplication of a relationship between a pair of KEs was taken into account when calculating the network analytics.

\section{Network analysis}

The level of degree, betweenness centrality and eccentricity were chosen to characterise the derived AOP network analytically due to their ability to quantify the position of a KE in relation to its neighbour KEs in the network using Cytoscape NetworkAnalyzer 3.3.2 App. Level of degree allowed for the identification of points of convergence and divergence, and to analyse the overall connectivity of the 
KEs across the AOP network, while the most upstream and downstream KEs across the AOP network were assessed on the basis of betweenness centrality and eccentricity. KEs with a higher score for the degree and betweenness centrality and a lower score for the eccentricity were considered the most common/highly connected KEs. Comparative analysis of multiple parameters provided the centrality score more efficiently and, therefore, less uncertainty in defining the most common/highly connected KEs.

\section{Results and discussion}

\section{Development of the AOP network for neurotoxicity}

Published AOPs in the AOP-Wiki were used to develop a derived AOP network for human neurotoxicity. Initially, 12 linear AOPs relevant to human neurotoxicity were identified in accordance with the methodology (Fig. 1) outlined in "Materials and methods". Table 1 provides details of the AOPs selected including the stage of their development at the time of retrieval.

After curation, with the exception of AOPs IDs 10, 26, and 152, all the nine other AOPs were found to share common KEs and were mapped in a network. The developed AOP network is shown graphically in Fig. 2. The MIE defined as binding of the antagonist NMDA receptors is common to two AOPs (AOP ID 12 and AOP ID 13) and similarly the MIE inhibition of $\mathrm{Na}+/ \mathrm{I}-$ symporter (NIS) linked two other AOPs (AOP ID 54 and AOP ID 134). Three AOs defined as neurodegeneration, Parkinsonian motor deficits and impairment of learning and memory/decrease of cognitive function connected all nine AOPs.

The common KEs across the network are represented by the reduction of the human brain-derived neurotrophic factor (BDNF), mitochondrial dysfunction, oxidative stress, neuroinflammation, cellular injury/death, degeneration of dopaminergic neurons of the nigrostriatal pathway, decrease of neuronal network function, decrease in the synthesis of the thyroid hormones (TH), decrease in thyroxine (T4) in serum and neuronal tissue. Interestingly, different upstream KEs contribute to the same common KEs triggering different downstream KEs. For example, oxidative stress is initiated by two MIEs: activation of CYP2E1 and binding to SH/ $\mathrm{SeH}$ proteins of two different AOPs. At the same time, once triggered, oxidative stress leads to several other downstream KEs, such as dyshomeostatis of glutamate, lipid peroxidation and unfolded protein response.

The most centrally located KE across the network is cell injury/death triggered by several mechanisms. For instance, reduced levels of BDNF, which is widely expressed in the developing and mature central nervous system (CNS), cause abberations in neuronal morphology and function, including neuronal cell death, since as a neurotrophic factor, it plays an important role in neuronal survival, proliferation, differentiation (synaptogenesis) and maturation (AOP ID 13). Another crucial KE is neuroinflammation which triggers cell injury/death and neurodegeneration through the increased release of different pro-inflammatory mediators from activated microglia and astrocytes exacerbating neurodegeneration which potentiates neuroinflammation (AOP ID 17). Therefore, cell injury/death is involved in a feedback loop mechanism of cellular injury/death-neuroinflammationneurodegeneration. Impaired proteostatis through the disregulation of the ubiquitin-proteasome system (UPS) and the autophagy-lysosome pathway (ALP) increases accumulation of certain proteins (e.g. $\alpha$-synuclein), contributing to the degeneration of dopaminergic neurons of the nigrostriatal pathway that further leads to the motor deficits observed in Parkinson's disease (AOP ID 3). Cell injury/death also leads directly to the decrease of the neuronal network function implied in the impairment of learning and memory/decrease of cognitive function (AOPs IDs 13, 17, 48, 54). Dyshomeostatis of glutamate and mitochondrial dysfunction are other mechanisms associated with neuronal cell injury/death (see references for each AOP in AOP-Wiki).

The AOP network for neurotoxicity relies solely on KERs established between adjacent KEs. The use of the adjacent relationships between KEs shows the biological plausibility of triggering neurodegeneration as one of the most common/converging KEs (AOP ID 48) and AOs (AOP ID 12 and AOP ID 260) through different signalling pathways. Depending on the brain structure and the sub-type of neurons undergoing neurodegeneration, different AOs can be triggered. Indeed, as illustrated through this network, degeneration of dopaminergic neurons (DA) in substantia nigra (SN) leads to motor deficit, the AO in Parkinson's disease (AOP ID 3). However, neurodegeneration in the hippocampus or cortex leads mainly to impairment of learning and memory/decrease of cognitive function (AO of AOPs IDs $12,13,17,42,48,54,134)$.

The empirical evidence supporting KERs in this AOP network is mainly described in a qualitative or semi-quantitative manner. The weight of evidence (WoE) supporting KERs varies from low to high, with low WoE possibly based on poor empirical data or contradictory information. To increase the empirical evidence supporting the KER, more experiments designed for such purpose may be required. In addition, to improve confidence in an AOP network, for instance to support regulatory use (e.g. risk assessment), a better quantitative definition of the thresholds to trigger respective KEs within each KER is needed. In other words, the availability of quantitative KERs should enable an assessment of the likelihood, and under what conditions of chemical concentration and exposure duration, a cascade of key events triggered by an MIE will lead to an AO. 


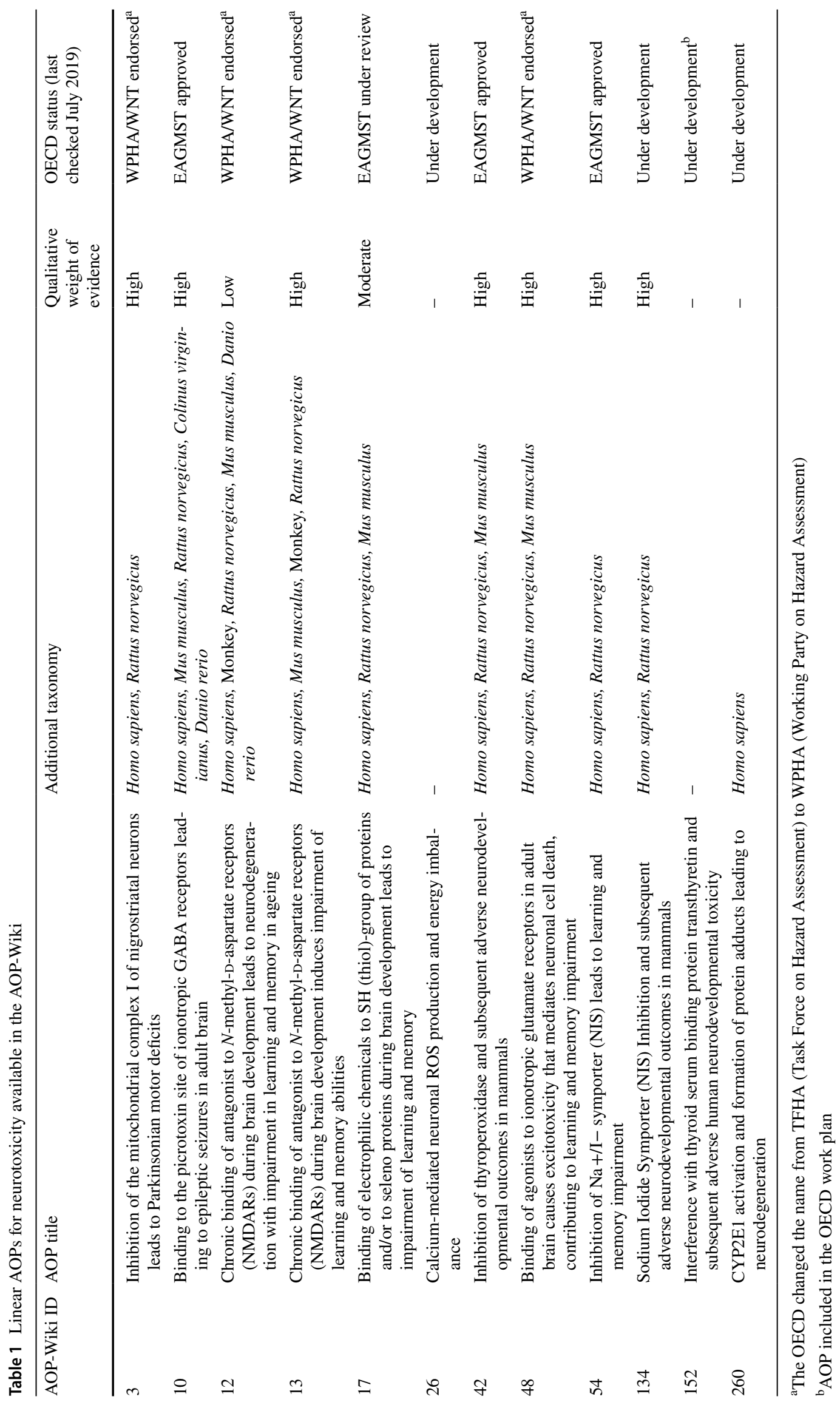


Table 2 The list of identified seven convergent and 12 divergent KEs

\begin{tabular}{ll}
\hline KE type & KE title \\
\hline Convergent key events & \\
KE & General apoptosis \\
AO & Impairment of learning and memory/cognitive function, decreased \\
KE & Neuroinflammation \\
AO & Parkinsonian motor deficits \\
KE & Synaptogenesis, decreased \\
KE & Tissue resident cell activation \\
KE & Unfolded protein response \\
Divergent key events & \\
KE & BDNF, reduced \\
MIE & Binding of agonist, ionotropic glutamate receptors \\
MIE & Binding of antagonist, NMDA receptors \\
MIE & Binding of inhibitor, NADH-ubiquinone oxidoreductase (complex I) \\
MIE & Binding, SH/SeH proteins involved in protection against oxidative stress \\
KE & Cell injury/death \\
MIE & CYP2E1 activation \\
KE & Increased pro-inflammatory mediators \\
MIE & Inhibition, Na +/I- symporter (NIS) \\
KE & Lipid peroxidation \\
KE & Oxidative stress \\
MIE & Thyroperoxidase, inhibition \\
\hline
\end{tabular}

\section{Analytical characterisation of the AOP network for neurotoxicity}

The analysis was performed on the derived AOP network for neurotoxicity that contains KEs and adjacent relationships between KEs of nine linear AOPs. As a result, network analytics confirmed that the most hyperlinked KE across the network is cell injury/death, followed by neuroinflammation, neurodegeneration, decrease in neuronal network function, and reduction of BDNF, with a level of degree of 13, 10, 8,8 and 8 , respectively. The least connected KEs were the MIEs with a level of degree 1, such as binding of agonist to the ionotropic glutamate receptors. The AO of Parkinsonian motor deficit also has a level of degree of 1 , as only one linear AOP is currently developed for this AO. The overall connectivity of the KEs is shown in Fig. 3.

The level of degree of KEs helped to identify points of convergence (common KEs) and divergence across the network, as listed in Table 2. Seven convergent KEs and twelve divergent KEs were identified following the score of the in-degree and out-degree. Points of convergence are defined as KEs linked to more upstream than downstream KEs (Villeneuve et al. 2018a), while points of divergence are defined as KEs linked to more downstream than upstream KEs (Villeneuve et al. 2018a). For example, oxidative stress is linked to three downstream KEs (glutamate dyshomeostasis, unfolded protein response, lipid peroxidation) and two upstream KEs defined as MIEs (CYP2E1 activation, binding to $\mathrm{SH} / \mathrm{SeH}$ proteins). The $\mathrm{AO}$ of the impairment of learning and memory/decrease of cognitive function has the highest number of incoming KERs (in-degree) with a score of seven, while the highest number of outgoing KERs (out-degree) is cell injury/death with a score of seven. This indicates that the impairment of learning and memory/decrease of cognitive function is a point of high convergence and cell injury/ death is a point of high divergence. Furthermore, all the MIEs of the network were identified as points of divergence, except the MIE protein adduct formation described in the AOP ID 260, which was linked to one upstream and one downstream KE. The protein adduct formation leads to the accumulation of unfolded proteins in the endoplasmic reticulum (downstream KE), but also lipid peroxidation (upstream $\mathrm{KE}$ ) contributes to the formation of protein adducts through one of its main products 4-hydroxynonenal (https://aopwi ki.org/aops/260).

Another important analytical measure is the eccentricity, which is a node centrality index that helps to sort the KEs into upstream and downstream KEs. A low score of eccentricity shows that the KE is more centrally located within the network and can be easily influenced by other KEs with which it is interconnected. The most centrally located KE, according to the eccentricity, is the reduction of BDNF with a score of 5. The most upstream KEs, according to their eccentricity, are the two MIEs: inhibition, $\mathrm{Na}+/ \mathrm{I}-$ symporter (NIS) and binding of agonist, Ionotropic glutamate receptors, with a score of 10 which indicates the maximum 


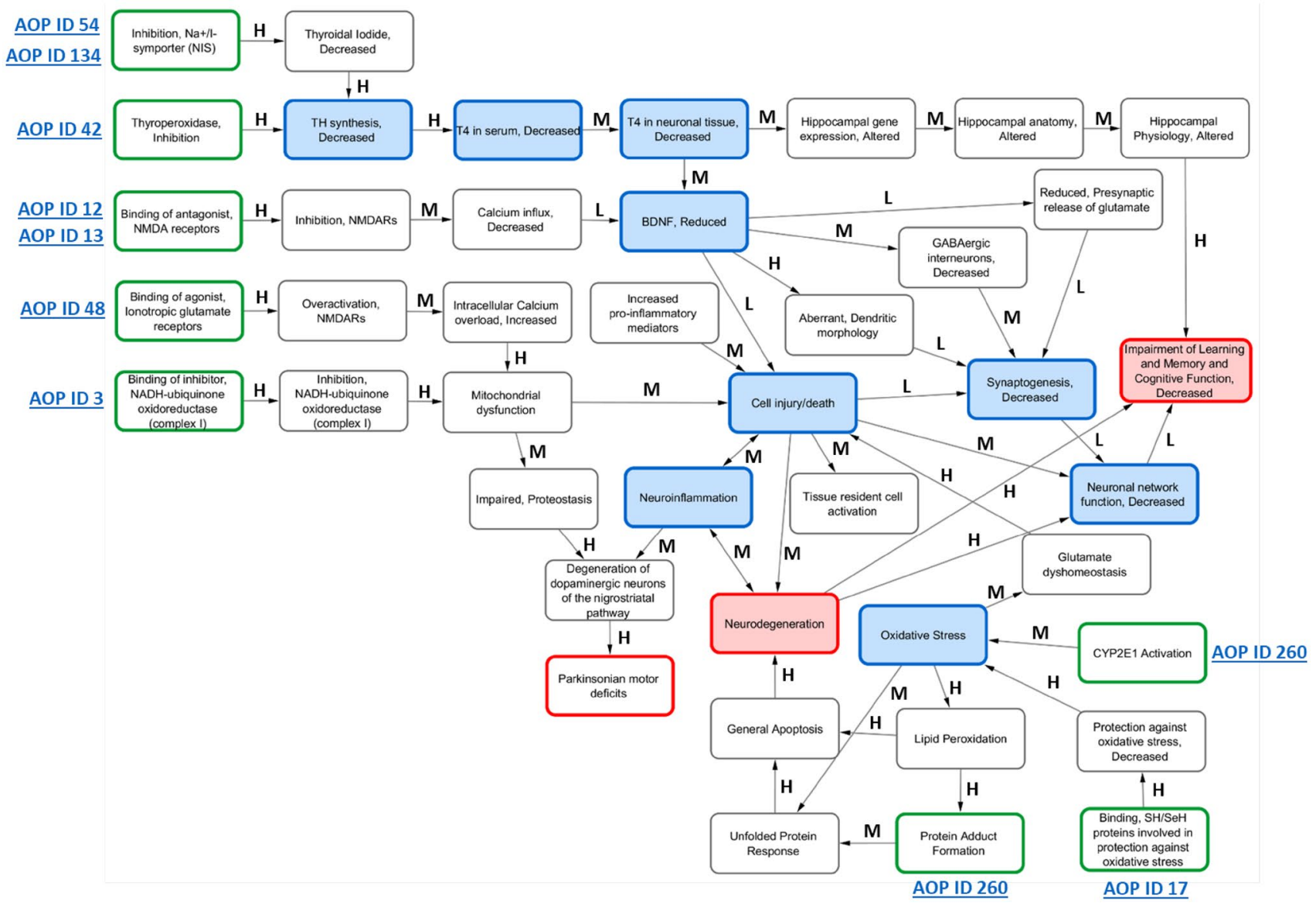

Fig. 2 Derived network of nine AOPs for neurotoxicity containing adjacent key event relationships. Green squares indicate an MIE, red squares indicate an $\mathrm{AO}$, and blue squares indicate the most common/ highly connected KEs. Solid arrows indicate relationships between KEs that are adjacent. The qualitative weight of evidence between

distance to the other KEs. These results are represented as a heat map in Figure S1 of the supplementary material.

Betweenness centrality measures the number of shortest paths between any two KEs in the AOP network that passes through the KE of interest (Villeneuve et al. 2018a). The $\mathrm{KE}$ with the highest betweenness centrality score was cell injury/death, which means that it is located most centrally within the network and confirms the assumptions made based on the graphical representation. This information complements the results given by the level of degree. These results are represented as a heat map in Figure S2 of the supplementary material.

The statistical distribution of the number of KEs, in relation to the level of in-degree and out-degree, shows that the majority of the KEs are associated with at least other two KEs, with almost $57 \%$ of KEs for the in-degree and almost $72 \%$ for the out-degree (Figure S3A and B). The number of shared AOPs by a KE varies between one and nine AOPs (Figure S3C). This has a tremendous impact on the two KEs is annotated as $\mathrm{H}$ for high, $\mathrm{M}$ for medium and $\mathrm{L}$ for low level of evidence. The KE cell injury/death is a common KE across the AOP network, being the most centrally located and most highly connected (colour figure online)

development and analysis of an AOP network, as a network can be modelled once a KE shares at least two linear AOPs. The eccentricity parameter reveals that almost $67 \%$ of the KEs are so interconnected that they cannot be categorised as upstream or downstream KEs (Figure S3D). The common KEs identified based on both the graphical representation and analytics of the AOP network for neurotoxicity could serve as a basis for developing/selecting in vitro assays. These in vitro test methods could be included in an Integrated Approach to Testing and Assessment (IATA) for evaluating neurotoxicity induced by individual chemicals and mixtures (Bal-Price and Meek 2017). For example, Li et al. (2019) propose an IATA for the assessment of developmental neurotoxicity by selecting a set of assays that can be used to assess common KEs. Our work also supports the common KEs identified by Li et al. (2019) as testing endpoints. Such IATA offer the possibility of addressing different regulatory needs including screening and prioritisation, hazard identification/characterisation or even risk assessment if combined 


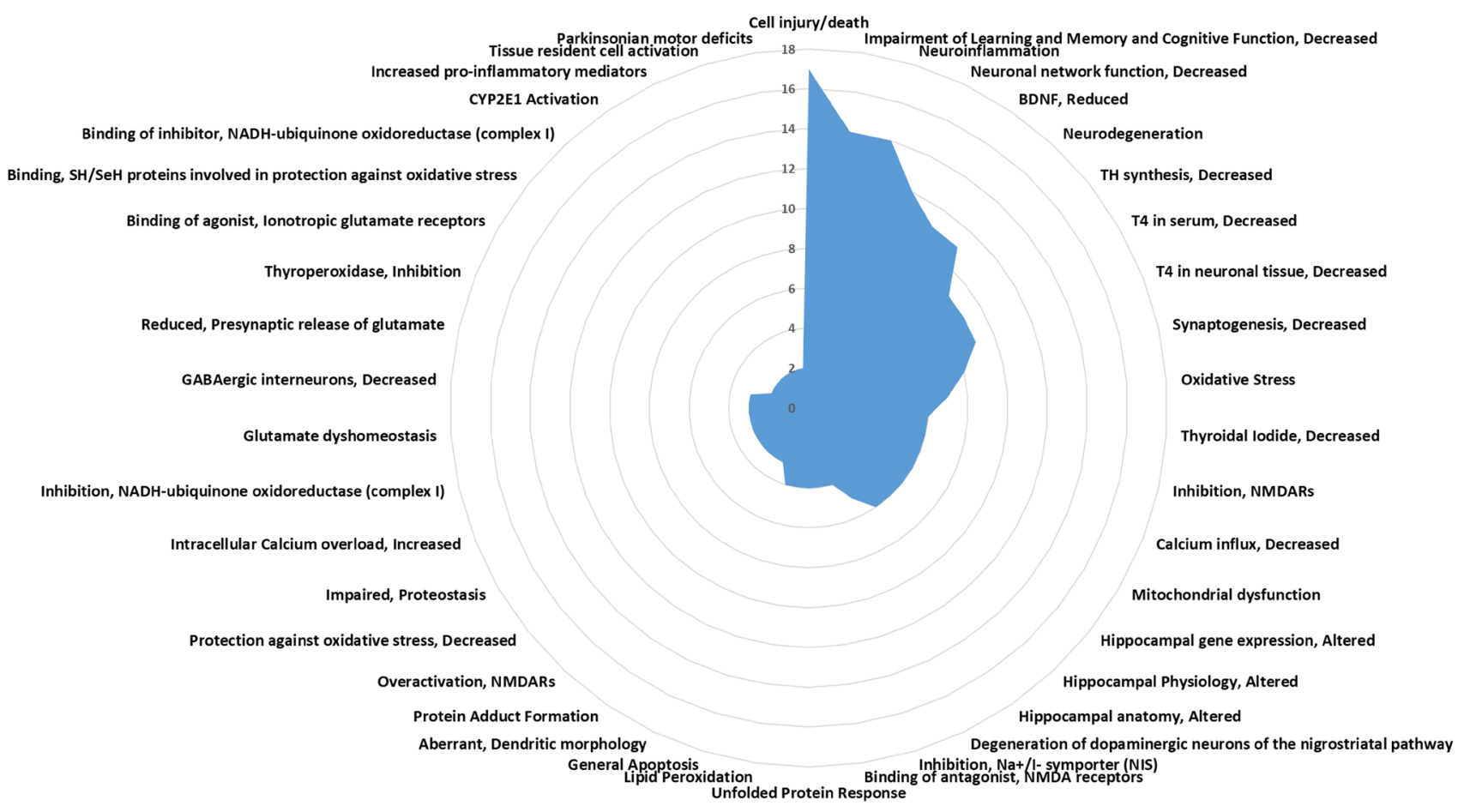

Fig. 3 The overall connectivity of KEs used to develop the derived AOP network for neurotoxicity. The score indicates the number of the KERs associated with a KE. Cell injury/death has the highest score, which means that it is the most interconnected KE across the

with exposure and ADME data (Aschner et al. 2017; BalPrice et al. 2018a, b).

\section{Adjacency and non-adjacency in the context of the AOP network for neurotoxicity}

One aspect considered while developing the AOP network was the inclusion of solely adjacent relationships between KEs. Relying only on directly connected KEs facilitates the quantitative simulation of the AOP network. However, the topological difference between AOPs networks consisting of adjacent and both adjacent and non-adjacent relationships was evaluated by comparing the analytical parameters of the two AOP networks. The AOP networks containing both types of interactions are represented graphically in Figure S4 in the supplementary material.

Four out of nine AOPs included in the AOP network contain solely adjacent relationships (AOPs IDs 12, 13, 48, and 260 ) and five of nine AOPs contain both types of relationships (AOPs IDs 3,17,42, 54, and 134). Several KEs were involved in non-adjacent relationships, including reduction of BDNF, decrease in TH synthesis, and decrease of T4 in the serum. Cell injury/death and reduction of BDNF remain the most connected/common KEs across the network. The $\mathrm{AO}$ defined as impairment of learning and memory/decrease network. The least connected KE was the AO of Parkinson's motor deficits due to the fact that only one AOP is currently available for this outcome

of cognitive function is involved in five non-adjacent relationships, besides the other seven adjacent relationships and, therefore, becomes the most connected KE across the network.

Since a non-adjacent relationship is likely to be associated with more biological processes, an AOP network containing both types of relationships implies more connections, representing a higher level of biological complexity. Network analytics show differences in terms of the distance and path length. At the same time, a given stressor might trigger all kinds of relationships and the AOP network serves as a suitable platform for such evaluations. For the initial development of a quantitative AOP (qAOP), it is easier to model adjacent KEs only rather than include both types. However, this also depends on data availability and the scope of the quantitative modelling.

\section{Mapping stressors to the AOP network}

Chemicals either individually or in combinations (mixtures), as well as other factors such as particles and infectious agents, represent stressors responsible for the initiation of an AOP (OECD 2017). Mapping stressors to linear AOPs in an AOP network allows for the evaluation of interactions between co-occurring stressors. Furthermore, 
for the purpose of an IATA, it is essential to derive a clear relationship between MIEs and AOs and whether there are interactions between AOPs (OECD 2016). Different (types of) stressors may interact at the MIE or at downstream KEs common to multiple AOPs.

The AOP network for neurotoxicity is represented by chemicals as stressors, with no additional types being mentioned in the AOP-Wiki module. Based on an understanding of the nature of MIEs, in silico models can be derived and, as a result, inform IATA and read-across. For example, several types of MIEs with associated AOPs have been distinguished and described by Cronin and Richarz (2017), including covalent reactivity, changes in receptor or enzyme activity. The different types of MIEs are identified in the AOP network for neurotoxicity including chronic receptor inhibition (binding of antagonist to NMDA receptors) and activation (binding of agonist to ionotropic glutamate receptors, binding of inhibitor to NADH-ubiquinone oxidoreductase (complex I), binding to $\mathrm{SH} / \mathrm{SeH}$ proteins involved in the protection against oxidative stress, inhibition of thyroperoxidase, inhibition of $\mathrm{Na}+/ \mathrm{I}-$ symporter (NIS)), covalent reactivity (protein adduct formation) and enzyme activation (CYP2E1 activation). These examples could serve as starting points in the development of in silico models for neurotoxicity.

AOP networks are critical for addressing exposures to multiple stressors that lead to the same AO or to individual stressors that perturb multiple MIEs (Knapen et al. 2015; Villeneuve et al. 2018a, b). For example, the inhibition of thyroperoxidase (MIE ID 279) is induced by chemicals with industrial and therapeutic uses, such as antifungal agents (e.g. 2(3H)-benzothiazolethione, mercaptobenzothiazole), antithyroid agents (e.g. thiouracil, propylthiouracil, methimazole), pesticides (e.g. ethylenethiourea), industrial agents (e.g. 4-nonylphenol) and cosmetic ingredients (e.g. resorcinol). On the other hand, acrylamide, with multiple chemical and industrial applications, a widely occurring food contaminant from cooking, binds to SH/Seleno proteins, an MIE in the AOP ID 17 that leads to the impairment in learning and memory through neuronal degeneration. Acrylamide also induces protein adduct formation, an MIE in AOP ID 260, that leads to neurodegeneration, an AO in AOPs IDs 12 and 260 and a KE in AOP ID 48. This is because of the electrophilic nature of acrylamide that covalently reacts with nucleophilic sulfhydryl groups on certain proteins that are critically involved in membrane fusion of the nerve terminals (LoPachin 2004; Lopachin and Decaprio 2005).

For qAOP modelling purposes, in its initial phase of development, evidence that a chemical can induce an entire AOP is of great help. However, the AOPs evaluated herein lacked stressors known to be active across all the biological levels of the AOP. This might be due to the fact that no compounds were yet tested for those AOPs or were not tested at high enough concentrations. The only AOP that has chemicals associated with all KEs is AOP ID 42 "Inhibition of Thyroperoxidase and Subsequent Adverse Neurodevelopmental Outcomes in Mammals". Therefore, a qAOP could be derived and was modelled by Hassan et al. (2017) for 6-propyl-2-thiouracil which is an enzyme inhibitor that is known to trigger AOP ID 42. Information on all chemicals collected for the AOPs used for modelling the AOP network is provided in the supplementary material.

The current description of stressors in the AOP-Wiki is lacking in detail, so it would be very valuable to include more information such as mechanistic knowledge related to the kinetics, existing QSAR models and read-across predictions, as well as other data sources. This would make the AOP-Wiki module not only a repository, but also a resource for modelling qAOPs.

One of the critical requirements for in silico modelling is the availability of reliable data. A data repository that could be used for in silico modelling is the ToxCast ${ }^{\mathrm{TM}}$ dashboard. It contains 76 assays studied on the brain tissue for several endpoints, including oxidative stress, binding to dopaminergic or GABAergic neurons that characterise KEs of a linear AOP. Following the AOPs currently published in the AOP-Wiki KB, 15 assays were identified as applicable to the AOPs known to induce neurotoxicity and that could be used to inform the AOP network. The results of linking assays to KEs of the AOP network are listed in Table S1 in the supplementary material.

\section{The use of AOPXplorer}

The AOPXplorer module of the AOP-Wiki KB was designed to visualise and explore AOP networks for a given adverse outcome. It also allows the uploading of additional data, e.g. high-throughput screening, omics and dose-response data that can be used to predict the adverse outcome. The AOPXplorer was developed as an App of the Cytoscape software that can be easily downloaded and installed. This facilitates the development of AOP networks while making them living documents. Currently, the AOPXplorer repository contains 18 AOP networks developed for different endpoints, including coagulopathy, ulcer gastric, steatosis, lung fibrosis, skin sensitisation, epilepsy. The AOP network modelled herein was also included in the AOPXplorer repository (Burgoon 2019). This allows further improvement and refinement of the AOP network by the scientific community that can view and further enrich the AOP network, thereby contributing to a better assessment of neurotoxicity.

\section{Applications of the derived AOP network}

The development of AOP networks is still in its infancy, but there are already examples of AOP networks applied 
to both human and other species toxicological endpoints as listed in the Table 3.

Because of its advantages, such as the use of analytics to characterise the position of a KE (including MIE and AO) within a network, the concept of a derived AOP network has a plethora of applications in predictive toxicology which are exemplified below.

\section{Types of modelling approaches for qAOPs}

The development and quantification of AOP networks are emergent topics in predictive toxicology and safety assessment. The Pellston Workshop (Hecker and LaLone 2019) addressed key challenges, approaches and solutions to progress the AOP framework, emphasising the need for more examples of where and how AOPs can fit in a regulatory context. Currently, three approaches have been proposed for modelling qAOPs: semiquantitative/quantitative weight of evidence (WoE) of qAOPs, probabilistic qAOP and qAOP networks, and mechanistic qAOPs (Perkins et al. 2019). Usually, quantification of WoE relies solely on tailored Bradford Hill considerations (Becker et al. 2017; Collier et al. 2016). However, ideally, the position of the KE within an AOP/ AOP network should also be considered. Another scientific workshop report (Kleinstreuer et al. 2016) proposed a mathematical approach to establish the relevance of KEs in $\mathrm{AO}$ prediction, based on the position of the event within the AOP and the available empirical data. Such details can be acquired from the analysis of topological parameters of KEs within an AOP/AOP network. This also allows for the reduction of the uncertainty of the final qAOP model.

However, it is challenging to move from the development and quantification of linear AOPs to the quantification of AOP networks. One approach is to apply Bayesian statistics to derive probabilistic of models. Several examples have already been published for human toxicological endpoints, such as chronic kidney injury (Zgheib et al. 2019), liver steatosis (Burgoon et al. 2017) and skin sensitisation (Jaworska et al. 2015). Furthermore, Bayesian networks can enable integration of multiple data types, including omics, toxicokinetics and toxicodynamics. The AOP network derived herein could be enriched with such data from in vitro studies (González-Ruiz et al. 2019; Schultz et al. 2015) to increase the confidence of the mechanistic understanding of the exposure to neurotoxicants.

Finally, mechanistic qAOPs encounter many challenges, one of them being lack of adequate and sufficient data. Foran et al. (2019) proposed a modular approach to develop a qAOP to take this limitation into account. They derived quantitative KERs for pairs of KEs for the AOP ID 48 (binding of agonists to ionotropic glutamate receptors in adult brain causes excitotoxicity that mediates neuronal cell death, contributing to learning and memory impairment). They quantified the relationship between the MIE (binding of agonist, ionotropic glutamate receptors) and KE2 (increased, intracellular calcium overload), the MIE (binding of agonist, ionotropic glutamate receptors) and KE4 (cell injury/death), and KE4 (cell injury/death) and AO (impairment, learning and memory). As this AOP is included in the derived AOP network herein, the analysis of the AOP network gives confidence in the modular approach to qAOP modelling: the neuronal cell injury/ death is one of the most common/highly connected KE across the network, while the increase in the intracellular calcium overload is an essential $\mathrm{KE}$ that leads to the reduction in BDNF.

Table 3 Summary of eight derived AOP networks currently developed and publicly available

\begin{tabular}{|c|c|c|c|}
\hline AOP network title & Taxonomy & Aim & Reference \\
\hline $\begin{array}{l}\text { AOP network related to reproductive and devel- } \\
\text { opmental toxicity in fish }\end{array}$ & Fish & Toxicity assay development & Knapen et al. (2015) \\
\hline $\begin{array}{l}\text { AOP network for disrupted androgen-and insulin- } \\
\text { like hormone } 3 \text { (INSL-3)-dependent in male rats }\end{array}$ & Rodents & Evaluation of chemical mixtures & Howdeshell et al. (2017) \\
\hline $\begin{array}{l}\text { AOP network linking activation of the nicotinic } \\
\text { acetylcholine receptor in honey bees to colony } \\
\text { death }\end{array}$ & Bees & $\begin{array}{l}\text { Evaluation of biological plausibility and empiri- } \\
\text { cal support to identify knowledge gaps }\end{array}$ & LaLone et al. (2017) \\
\hline $\begin{array}{l}\text { AOP network for metabolic disorders mediated by } \\
\text { hepatic steatosis }\end{array}$ & Homo sapiens & Identification of critical paths & Knapen et al. (2018) \\
\hline Decreased serum thyroid hormone AOP network & $\begin{array}{l}\text { Rodents } \\
\text { Amphibians } \\
\text { Fish }\end{array}$ & $\begin{array}{l}\text { The use of layers and the identification of points } \\
\text { of convergence/divergence }\end{array}$ & Knapen et al. (2018) \\
\hline Hub KEs for inflammation-related AOP network & Not specified & $\begin{array}{l}\text { Connection of AOPs that previously had no } \\
\text { shared KEs }\end{array}$ & Villeneuve et al. (2018b) \\
\hline Cytochrome P45019 [CYP19]-AOP network & Not specified & Linking all possible AOPs to an AOP & Villeneuve et al. (2018a) \\
\hline Thyroxine [T4]-AOP network & Not specified & Linking all possible AOPs to a process & Villeneuve et al. (2018a) \\
\hline
\end{tabular}




\section{Conclusions}

An AOP network for human neurotoxicity was developed using the principles of the derived AOP network. A workflow was formulated which can be adapted according to the purpose of the investigation. Even though the developed AOP network is simplistic and probably incomplete, the results provide a solid basis for prioritising the testing of KEs, for quantifying KEs and KERs, and for quantitative modelling of the AOP network. In addition, the work could be useful for identifying biomarkers of toxicity at different biological levels and for further developing in silico and in vitro test methods, thereby contributing to the assessment of neurotoxicity without animal testing (Bal-Price et al. 2017).

More generally, AOP networks strengthen the utility of the OECD AOP-KB by increasing scientific confidence in the application of individual AOPs, facilitating better understanding of their roles as individual blocks in the network of complex biological interactions. Network analytics can be utilised to analyse multiple perturbations and complex interactions across the biological and time scales of interconnected AOPs. As additional mechanistic details enrich the existing AOPs, it is envisaged that AOP networks will become more complete and informative for predictive toxicology and regulatory decision making.

There are several challenges in the development of an AOP network:

1. The ontology annotations influence the construction of an AOP network. There are still KEs titled differently while having the same meaning and/or referring to the same process. For example, the AOP network on neurotoxicity contains KEs related to the mitochondrial dysfunction: KE ID 177, KE ID 1185, KE ID 1186. All of these KEs can be grouped or renamed under a common KE umbrella. This would also facilitate further quantification in terms of response-response relationships useful for systems toxicology. Following an expert review, such annotations can be easily amended. Slenter et al. (2018) evaluated the WikiPathways database (https ://www.wikipathways.org) in terms of the content and curation of metabolic pathways and showed the benefits of harmonising the annotation of metabolism and metabolic pathways.

2. As information on the compensatory mechanisms for neurotoxicity is missing and not included in the OECD AOP-Wiki pages, such networks do not represent the entire complexity of biological processes (feedback and feedforward loops, etc.) and research in this sense is urgently needed. Understanding possible compensatory mechanism and adaptive changes, which take place ear- lier before the first $\mathrm{KE}$ is triggered, may moderate and contribute to the observed adverse outcome. If compensatory and adaptive mechanisms are effective, the cell is coping and toxicity is not taking place. At present, this kind of information is captured in the KER descriptions, but perhaps the AOP template should also be modified permitting visualisation and description of these processes.

3. The uncertainty of the network model partly arises from the stage of development of an AOP. An AOP network is built on individual AOPs that ideally follow the OECD requirements, hence giving confidence to the use of an AOP network for predicting toxicity and assessing chemical safety. Even though the majority of the individual AOPs published in the AOP-Wiki module are under development (almost two-thirds at the time of preparation of this manuscript), this should not limit their use as the basis of an AOP network. For example, four out of the initial 12 AOPs collected for the development of the AOP network for neurotoxicity were under development, from which one AOP was included in the OECD work plan. Therefore, it will be increasingly possible to develop AOP networks once the OECD AOP-KB becomes more populated with linear AOPs and associated mechanistic information.

4. When a linear AOP is updated, the AOP network should also include the changes. The AOPXplorer serves as a tool that can encompass such changes.

5. There are "orphan" AOPs that were not linked to the AOP network for neurotoxicity (AOPs IDs 10, 26, 152) and were, therefore, excluded in the current work. However, these may eventually provide additional KEs and KERs for expanding the network.

6. AOP networks can be derived for different applications, including development of toxicity assays (Knapen et al. 2015), evaluation of chemicals mixture (Howdeshell et al. 2017), evaluation of biological plausibility and empirical support to identify knowledge gaps (LaLone et al. 2017), etc. Herein, linear AOPs were investigated for the adverse outcome of developmental and adult/ ageing neurotoxicity induced in Homo sapiens.

7. To maximise the application of AOP networks, the use of network analytics provides an essential instrument for characterising the network and identifying common KEs and KERs. Several parameters defined by Villeneuve et al. (2018a), including level of degree, betweenness centrality and eccentricity, were applied in this work. Information regarding the centrality and connectivity of a KE, the most upstream or downstream KEs across the network, is of a great value for finding gaps in knowledge and unforeseen paths. The choice of metric(s) depends on the intended purpose, e.g. development of a battery of in vitro tests. 
8. The concept of AOP network should not be confused with the concept of a hub. A hub consists of several KEs closely linked and involved in the same biological process. Therefore, a hub can be part of an AOP network. For example, one of the common KEs identified was neuroinflammation. However, increase of proinflammatory mediators is also known to contribute to the cell apoptosis and necrosis, as AOP ID 17 illustrates. Since inflammation is a complex process, a key event describing it might not be identified as a connected node across the network as is the case of the increased proinflammatory mediators. Villeneuve et al. (2018b) developed a hub which links different MIEs to distinct inflammation-mediated AOs or to AOPs where inflammation is an essential exacerbating element. Such a hub allows interconnectivity with other AOPs that were previously disconnected, independent of the tissue.

Acknowledgements The funding of the European Union Marie Skłodowska-Curie Action Innovative Training Network in3 Project (http://estiv.org/in3/), under Grant No. 721975, is gratefully acknowledged. The authors of the AOPs used to derive the AOP network are also gratefully acknowledged. NS was a JRC trainee from September 2018 to February 2019. We would like to thank Lyle D. Burgoon for helping with the publication of the AOP network in the AOPXplorer App.

\section{Compliance with ethical standards}

Conflict of interest The authors declare that they have no conflict of interest.

Open Access This article is distributed under the terms of the Creative Commons Attribution 4.0 International License (http://creativeco mmons.org/licenses/by/4.0/), which permits unrestricted use, distribution, and reproduction in any medium, provided you give appropriate credit to the original author(s) and the source, provide a link to the Creative Commons license, and indicate if changes were made.

\section{References}

Aguayo-Orozco A, Taboureau O, Brunak S (2019) The use of systems biology in chemical risk assessment. Curr Opin Toxicol. https:// doi.org/10.1016/j.cotox.2019.03.003

Ankley GT, Bennett RS, Erickson RJ et al (2010) Adverse outcome pathways: a conceptual framework to support ecotoxicology research and risk assessment. Environ Toxicol Chem 29(3):730741. https://doi.org/10.1002/etc.34

Aschner M, Ceccatelli S, Daneshian M et al (2017) Reference compounds for alternative test methods to indicate developmental neurotoxicity (DNT) potential of chemicals: example lists and criteria for their selection and use. Altex 34(1):49-74. https://doi. org/10.14573/altex.1604201

Assenov Y, Ramírez F, Schelhorn S-E, Lengauer T, Albrecht M (2008) Computing topological parameters of biological networks.
Bioinformatics 24(2):282-284. https://doi.org/10.1093/bioin formatics/btm554

Bal-Price A, Meek MEB (2017) Adverse outcome pathways: application to enhance mechanistic understanding of neurotoxicity. Pharmacol Ther 179:84-95. https://doi.org/10.1016/j.pharmthera 2017.05.006

Bal-Price A, Crofton KM, Leist M et al (2015a) International STakeholder NETwork (ISTNET): creating a developmental neurotoxicity (DNT) testing road map for regulatory purposes. Arch Toxicol 89(2):269-287. https://doi.org/10.1007/s00204-015-1464-2

Bal-Price A, Crofton KM, Sachana M et al (2015b) Putative adverse outcome pathways relevant to neurotoxicity. Crit Rev Toxicol 45(1):83-91. https://doi.org/10.3109/10408444.2014.981331

Bal-Price A, Lein PJ, Keil KP et al (2017) Developing and applying the adverse outcome pathway concept for understanding and predicting neurotoxicity. Neurotoxicology 59:240-255. https://doi. org/10.1016/j.neuro.2016.05.010

Bal-Price A, Hogberg HT, Crofton KM et al (2018a) Recommendation on test readiness criteria for new approach methods in toxicology: exemplified for developmental neurotoxicity. Altex 35(3):306352. https://doi.org/10.14573/altex.1712081

Bal-Price A, Pistollato F, Sachana M, Bopp SK, Munn S, Worth A (2018b) Strategies to improve the regulatory assessment of developmental neurotoxicity (DNT) using in vitro methods. Toxicol Appl Pharmacol 354:7-18. https://doi.org/10.1016/j. taap.2018.02.008

Barabasi AL, Oltvai ZN (2004) Network biology: understanding the cell's functional organization. Nat Rev Genet 5(2):101-113. https ://doi.org/10.1038/nrg1272

Bassett DS, Sporns O (2017) Network neuroscience. Nat Neurosci 20(3):353-364. https://doi.org/10.1038/nn.4502

Becker RA, Dellarco V, Seed J et al (2017) Quantitative weight of evidence to assess confidence in potential modes of action. Regul Toxicol Pharmacol 86:205-220. https://doi.org/10.1016/j.yrtph .2017.02.017

Brandes U, Robins G, McCranie ANN, Wasserman S (2013) What is network science? Netw Sci 1(1):1-15. https://doi.org/10.1017/ nws. 2013.2

Burgoon DL (2019) AOPXplorer. http://apps.cytoscape.org/apps/aopxp lorer. Accessed 8 May 2019

Burgoon LD, Druwe IL, Painter K, Yost EE (2017) Using in vitro high-throughput screening data for predicting benzo[k]fluoranthene human health hazards. Risk Anal 37(2):280-290. https:// doi.org/10.1111/risa.12613

Collier ZA, Gust KA, Gonzalez-Morales B et al (2016) A weight of evidence assessment approach for adverse outcome pathways. Regul Toxicol Pharmacol 75:46-57. https://doi.org/10.1016/j. yrtph.2015.12.014

Cronin MTD, Richarz A-N (2017) Relationship between adverse outcome pathways and chemistry-based in silico models to predict toxicity. Appl Vitr Toxicol 3(4):286-297. https://doi.org/10.1089/ aivt.2017.0021

Edwards SW, Tan YM, Villeneuve DL, Meek ME, McQueen CA (2016) Adverse outcome pathways-organizing toxicological information to improve decision making. J Pharmacol Exp Ther 356(1):170-181. https://doi.org/10.1124/jpet.115.228239

Foran CM, Rycroft T, Keisler J, Perkins EJ, Linkov I, Garcia-Reyero N (2019) A modular approach for assembly of quantitative adverse outcome pathways. Altex. https://doi.org/10.14573/altex.1810181

Fritsche E, Grandjean P, Crofton KM et al (2018) Consensus statement on the need for innovation, transition and implementation of developmental neurotoxicity (DNT) testing for regulatory purposes. Toxicol Appl Pharmacol 354:3-6. https://doi.org/10.1016/j. taap.2018.02.004

González-Ruiz V, Schvartz D, Sandström J et al (2019) An integrative multi-omics workflow to address multifactorial toxicology 
experiments. Metabolites 9(4):79. https://doi.org/10.3390/metab 09040079

Hartung T, FitzGerald RE, Jennings P et al (2017) Systems toxicology: real world applications and opportunities. Chem Res Toxicol 30(4):870-882. https://doi.org/10.1021/acs.chemrestox.7b00003

Hassan I, El-Masri H, Kosian PA, Ford J, Degitz SJ, Gilbert ME (2017) Neurodevelopment and thyroid hormone synthesis inhibition in the rat: quantitative understanding within the adverse outcome pathway framework. Toxicol Sci 160(1):57-73. https://doi. org/10.1093/toxsci/kfx 163

Hecker M, LaLone CA (2019) Adverse outcome pathways: moving from a scientific concept to an internationally accepted framework. Environ Toxicol Chem 38(6):1152-1163. https://doi. org/10.1002/etc. 4385

Howdeshell KL, Hotchkiss AK, Gray LE Jr (2017) Cumulative effects of antiandrogenic chemical mixtures and their relevance to human health risk assessment. Int J Hyg Environ Health 220(2 Pt A):179_ 188. https://doi.org/10.1016/j.ijheh.2016.11.007

Jaworska JS, Natsch A, Ryan C, Strickland J, Ashikaga T, Miyazawa M (2015) Bayesian integrated testing strategy (ITS) for skin sensitization potency assessment: a decision support system for quantitative weight of evidence and adaptive testing strategy. Arch Toxicol 89(12):2355-2383. https://doi.org/10.1007/s00204-015-1634-2

Kleinstreuer NC, Sullivan K, Allen D et al (2016) Adverse outcome pathways: from research to regulation scientific workshop report. Regul Toxicol Pharmacol 76:39-50. https://doi.org/10.1016/j. yrtph.2016.01.007

Knapen D, Vergauwen L, Villeneuve DL, Ankley GT (2015) The potential of AOP networks for reproductive and developmental toxicity assay development. Reprod Toxicol 56:52-55. https://doi. org/10.1016/j.reprotox.2015.04.003

Knapen D, Angrish MM, Fortin MC et al (2018) Adverse outcome pathway networks I: development and applications. Environ Toxicol Chem 37(6):1723-1733. https://doi.org/10.1002/etc.4125

Krewski D, Acosta D Jr, Andersen M et al (2010) Toxicity testing in the 21st century: a vision and a strategy. $\mathrm{J}$ Toxicol Environ Health B Crit Rev 13(2-4):51-138. https://doi.org/10.1080/10937 404.2010.483176

LaLone CA, Villeneuve DL, Wu-Smart J et al (2017) Weight of evidence evaluation of a network of adverse outcome pathways linking activation of the nicotinic acetylcholine receptor in honey bees to colony death. Sci Total Environ 584-585:751-775. https://doi. org/10.1016/j.scitotenv.2017.01.113

Li Z, Ivanov AA, Su R et al (2017) The OncoPPi network of cancerfocused protein-protein interactions to inform biological insights and therapeutic strategies. Nat Commun 8:14356. https://doi. org/10.1038/ncomms14356

Li J, Settivari R, LeBaron MJ, Marty MS (2019) An industry perspective: a streamlined screening strategy using alternative models for chemical assessment of developmental neurotoxicity. Neurotoxicology 73:17-30. https://doi.org/10.1016/j.neuro.2019.02.010

LoPachin RM (2004) The changing view of acrylamide neurotoxicity. Neurotoxicology 25(4):617-630. https://doi.org/10.1016/j.neuro 2004.01 .004

Lopachin RM, Decaprio AP (2005) Protein adduct formation as a molecular mechanism in neurotoxicity. Toxicol Sci 86(2):214225. https://doi.org/10.1093/toxsci/kfi197
OECD (2016) Guidance document for the use of adverse outcome pathways in developingintegrated approaches to testing and assessment (IATA). Series on Testing and Assessment, No. 260. (ENV/ $\mathrm{JM} / \mathrm{MONO}(2016) 67)$

OECD (2017) Revised guidance document on developing and assessing adverse outcome pathways (ENV/JM/MONO(2013)6)

Perkins EJ, Gayen K, Shoemaker JE et al (2019) Chemical hazard prediction and hypothesis testing using quantitative adverse outcome pathways. Altex 36(1):91-102. https://doi.org/10.14573/ altex. 1808241

Pollesch NL, Villeneuve DL, O'Brien JM (2019) Extracting and benchmarking emerging adverse outcome pathway knowledge. Toxicol Sci 168(2):349-364. https://doi.org/10.1093/toxsci/kfz006

Schmidt BZ, Lehmann M, Gutbier S et al (2017) In vitro acute and developmental neurotoxicity screening: an overview of cellular platforms and high-throughput technical possibilities. Arch Toxicol 91(1):1-33. https://doi.org/10.1007/s00204-016-1805-9

Schultz L, Zurich MG, Culot M et al (2015) Evaluation of drug-induced neurotoxicity based on metabolomics, proteomics and electrical activity measurements in complementary CNS in vitro models. Toxicol In Vitro 30(1 Pt A):138-165. https://doi.org/10.1016/j. tiv.2015.05.016

Slenter DN, Kutmon M, Hanspers K et al (2018) WikiPathways: a multifaceted pathway database bridging metabolomics to other omics research. Nucleic Acids Res 46(D1):D661-D667. https:// doi.org/10.1093/nar/gkx1064

Sturla SJ, Boobis AR, FitzGerald RE et al (2014) Systems toxicology: from basic research to risk assessment. Chem Res Toxicol 27(3):314-329. https://doi.org/10.1021/tx400410s

Villeneuve DL, Crump D, Garcia-Reyero N et al (2014a) Adverse outcome pathway (AOP) development I: strategies and principles. Toxicol Sci 142(2):312-320. https://doi.org/10.1093/toxsc i/kfu199

Villeneuve DL, Crump D, Garcia-Reyero N et al (2014b) Adverse outcome pathway development II: best practices. Toxicol Sci 142(2):321-330. https://doi.org/10.1093/toxsci/kfu200

Villeneuve DL, Angrish MM, Fortin MC et al (2018a) Adverse outcome pathway networks II: network analytics. Environ Toxicol Chem 37(6):1734-1748. https://doi.org/10.1002/etc.4124

Villeneuve DL, Landesmann B, Allavena P et al (2018b) Representing the process of inflammation as key events in adverse outcome pathways. Toxicol Sci 163(2):346-352. https://doi.org/10.1093/ toxsci/kfy047

Vinken M, Knapen D, Vergauwen L, Hengstler JG, Angrish M, Whelan M (2017) Adverse outcome pathways: a concise introduction for toxicologists. Arch Toxicol 91(11):3697-3707. https://doi. org/10.1007/s00204-017-2020-z

Zgheib E, Gao W, Limonciel A et al (2019) Application of three approaches for quantitative AOP development to renal toxicity. Comput Toxicol 11:1-13. https://doi.org/10.1016/j.comto x.2019.02.001

Publisher's Note Springer Nature remains neutral with regard to jurisdictional claims in published maps and institutional affiliations. 